\title{
Behaviour of vertically loaded plate anchors under sustained uplift
}

\author{
C. HAN*, D. WANG*, C. GAUDIN*, C. D. O’LOUGHLIN* and M. J. CASSIDY*
}

\begin{abstract}
The use of plate anchors in permanent moorings for deep-water floating structures requires a thorough understanding of their performance under sustained and cyclic loading. This paper addresses the former aspect through a series of centrifuge tests on vertically loaded plate anchors in normally consolidated kaolin clay, supported by large-deformation finite-element analyses. The centrifuge experiments, which included particle image velocimetry measurements to identify the conditions leading to anchor failure, demonstrate that sustained loading can be maintained indefinitely if the anchor displacement at application of the sustained loading does not exceed $40 \%$ of the displacement reached at monotonic failure. This corresponds to a sustained load that is about $88 \%$ of the monotonic capacity, as identified from the numerical analyses. An important observation from both the centrifuge tests and the numerical analyses was a gap at the base of the anchor that developed under moderate load levels when the vertical effective stress at the base of the anchor reached zero. Although this so-called breakaway condition is generally associated with a lower capacity factor and a heightened potential for anchor failure, the conditions leading to breakaway also allow for consolidation-induced strength increase at the top face of the anchor and the mobilisation of partially drained or drained soil strength, both of which maintain anchor stability.
\end{abstract}

KEYWORDS: anchors \& anchorages; centrifuge modelling; clays; consolidation; finite-element modelling; offshore engineering

\section{INTRODUCTION}

The capacity of offshore plate anchors under short-term monotonic undrained loading is generally well understood. For instance, the undrained capacity of suction embedded plate anchors (SEPLAs) has been investigated through largescale field tests (Wilde et al., 2001; DNV, 2002), small-scale laboratory or centrifuge tests (Rowe, 1978; Das \& Singh, 1994; Gaudin et al., 2006) and numerical modelling (Wang et al., 2010, 2013; Yang et al., 2010; Chen et al., 2013; Tho et al., 2013), and analytical force-resultant plasticity models have been developed to describe their load-displacement behaviour (Cassidy et al., 2012; Yang et al., 2012). However, there are uncertainties regarding the proportion of the available monotonic capacity that remains available during longterm sustained loading. This is a relevant design condition for plate anchors owing to the sustained loading generated by tides and seasonal currents. It is seeing an increased focus as large plate anchors, such as SEPLAs, are being considered as a taut-moored option for mooring permanent production facilities (Wong et al., 2012).

Experimental data presented by Wong et al. (2012) showed stable anchor behaviour when the magnitude of the sustained loading did not exceed $85 \%$ of the monotonic ultimate capacity. Above that level, the anchor experienced accelerating displacements, eventually leading to anchor failure. In this paper, a combination of centrifuge and numerical modelling is employed to show that anchor stability is controlled by three factors: $(a)$ the drainage conditions generated during sustained loading; $(b)$ consolidation-induced strength changes in the soil above the anchor; and (c) the formation of a gap at the base of the anchor.

Manuscript received 21 October 2015; revised manuscript accepted 26 February 2016. Published online ahead of print 12 April 2016.

Discussion on this paper closes on 1 January 2017, for further details see p. ii.

* Centre for Offshore Foundation Systems and ARC CoE for Geotechnical Science and Engineering, The University of Western Australia, Crawley, WA, Australia.

\section{ANCHOR CAPACITY IN CLAY}

The uplift capacity of plate anchors under long-term sustained loading, as shown in Fig. 1, requires an understanding of three main processes: $(a)$ the potential for breakaway at the base of the anchor; $(b)$ the plate velocity, which dictates if the mobilised strength is undrained, partially drained or drained; and $(c)$ any additional strength of the soil above the anchor associated with excess pore pressure dissipation. This section will review these processes and discuss their influence on anchor capacity.

\section{Predicting undrained capacity and potential breakaway}

The monotonic capacity of plate anchors in clay, $F_{\max }$, under undrained conditions is conventionally expressed as

$$
F_{\max }=N_{\mathrm{c}} A s_{\mathrm{u}}
$$

where $A$ is the projected area of the anchor, $s_{\mathrm{u}}$ is the undrained shear strength of the soil at the anchor embedment and $N_{\mathrm{c}}$ is a bearing capacity factor. Selection of $N_{\mathrm{c}}$ is controlled by assumptions made regarding soil flow around the anchor, and more particularly on the bonding conditions at the base of the anchor. Rowe \& Davis (1982) simplified the plate base-soil interaction as two extreme cases: no breakaway or immediate breakaway.

For the no-breakaway case, tensile total stresses at the base of the anchor (associated with negative excess pore pressures with respect to the hydrostatic pressure, also called suction) are sufficiently high such that the soil remains fully attached to the anchor base. The corresponding bearing capacity factor, $N_{\mathrm{c}}$, has been explored extensively as a function of the embedment depth using model tests (Gaudin et al., 2006; O'Loughlin et al., 2014), finite-element (FE) simulations (Thorne et al., 2004; Song et al., 2008; Wang et al., 2010, 2013; Tian et al., 2014; Wang \& O'Loughlin, 2014) and plasticity limit analyses (Yang et al., 2012). Collectively these studies show that $N_{\mathrm{c}}$ increases with embedment depth to a limiting value for deeply buried anchors, which is a function 


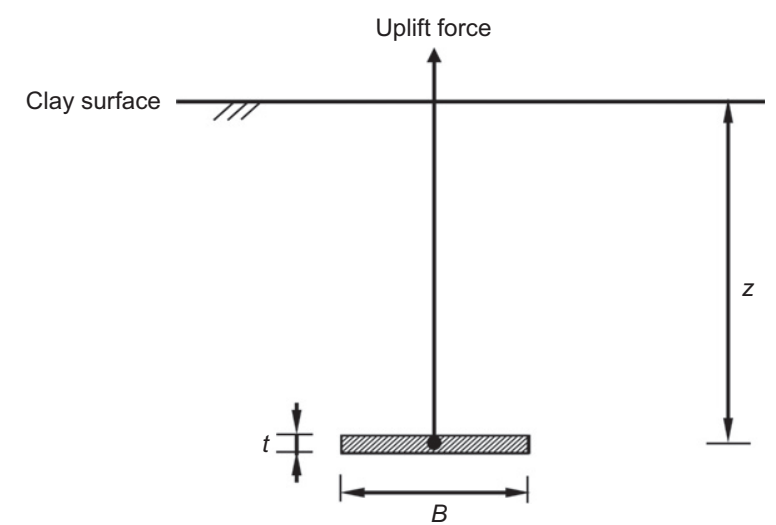

(a)

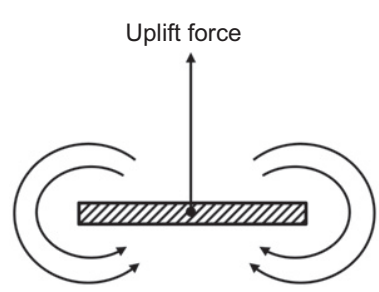

(b)

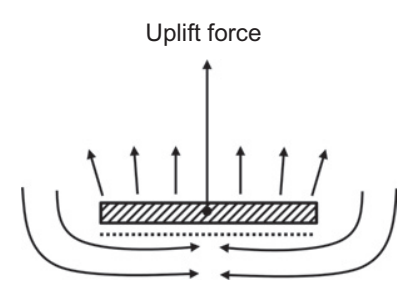

(c)
Fig. 1. Problem notation and description: (a) a deeply embedded strip plate anchor subjected to vertical loading; (b) no-breakaway condition; (c) breakaway condition

of anchor geometry, roughness and load inclination. The limiting $N_{\mathrm{c}}$ values reported in the literature for the deeply embedded strip plates considered in this paper are shown (as dashed lines) in Fig. 2, with the typical soil failure mechanism as shown in Fig. 1(b). The upper bound solution for an ultrathin plate is $N_{\mathrm{c}}=11 \cdot 42$, with minimal influence of plate roughness (Rowe, 1978), increasing to $N_{\mathrm{c}}=12 \cdot 1$ for a plate thickness ratio, $t / B=0 \cdot 14$ (O'Neill et al., 2003), where $B$ represents the plate breadth. This range encompasses findings from FE analyses reported by Elkhatib \& Randolph (2005), who showed that $N_{\mathrm{c}}$ increases from 11.58 at $t / B=0.05$ to $11 \cdot 78$ at $t / B=0 \cdot 14$. Det Norske Veritas (DNV) design guidelines recommend $N_{\mathrm{c}}=12$ for deeply buried strip anchors (DNV, 2002).

For the immediate breakaway case, no tensile total stresses are sustained at the base of the anchor, allowing the soil to separate from the anchor and a gap to form. Fig. 1(c) shows the failure mechanism for deeply embedded anchors with breakaway. Previous research using $1 \mathrm{~g}$ laboratory test (Rowe, 1978) and numerical approaches (Rowe \& Davis, 1982; Merifield et al., 2001; Song et al., 2008; Wang et al., 2010, 2013; Chen et al., 2013; Tho et al., 2013) found that the bearing capacity factor under immediate breakaway conditions depends on the overburden pressure and is always lower than that under no-breakaway conditions. For instance, bearing factors for a strip plate with $t / B=0 \cdot 05$, embedded in normally consolidated clay at $z=3 B$, increase from $N_{\mathrm{c}}=4 \cdot 5$ to $10 \cdot 8$, with increasing overburden pressure, compared with $N_{\mathrm{c}}=11.6$ for the no-breakaway case (Song et al., 2008).

\section{Drainage conditions as a function of anchor velocity}

Should the rate of loading be such that partially drained or drained conditions are generated, the mobilised soil strength will be higher than the undrained soil strength, $s_{\mathrm{u}}$. This trend has been well demonstrated in normally consolidated kaolin clay, through penetration resistance (e.g. House et al., 2001;

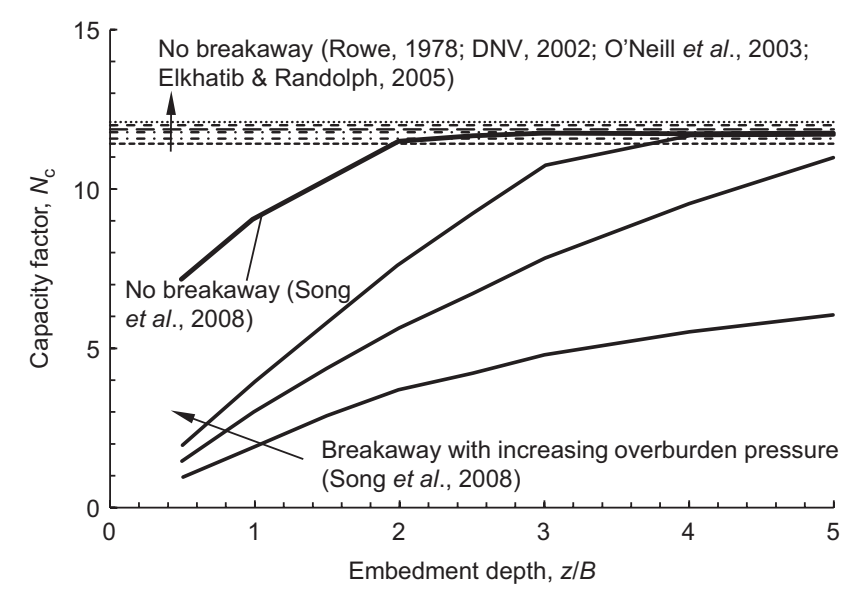

Fig. 2. Capacity factor of strip anchors with embedment depth for breakaway and no-breakaway cases (solid lines) and limiting values for deeply embedded no-breakaway case (dashed lines)

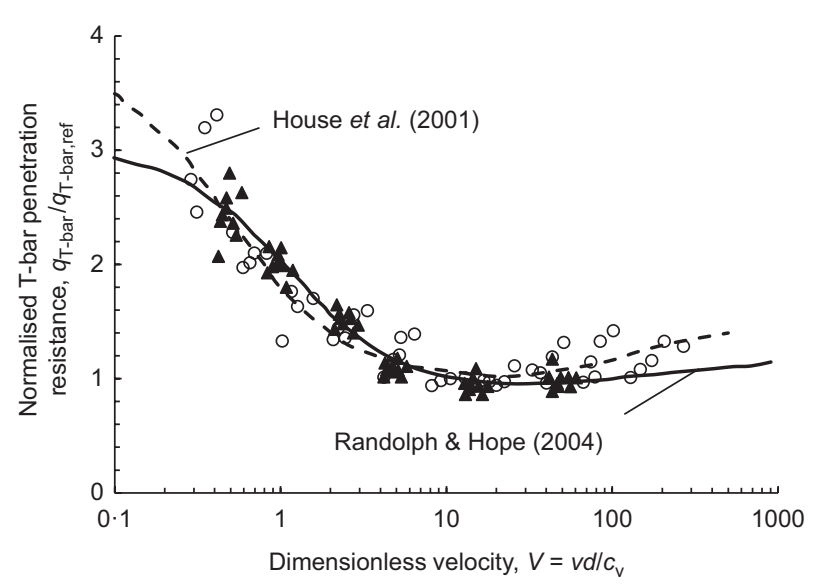

Fig. 3. Normalised T-bar resistance variation with dimensionless velocity

Randolph \& Hope, 2004; Lehane et al., 2009; Colreavy et al., 2016). This dependence of soil strength on drainage conditions is generally considered in terms of a dimensionless velocity, $V=v B / c_{\mathrm{v}}$, where $v$ is the (anchor) velocity. The response is typically as shown in Fig. 3 from House et al. (2001) and Randolph \& Hope (2004), which (for this example) plots the variation of T-bar resistance $\left(q_{\mathrm{T}-\mathrm{bar}}\right)$ normalised by the T-bar resistance mobilised during undrained conditions, $q_{\mathrm{T}-\mathrm{bar}, \mathrm{ref}}$, against $V=v d / c_{\mathrm{v}}$ (where for this example the T-bar diameter, $d$, is used in place of the plate breadth, $B$ ). A minimum penetration resistance, associated with mobilisation of an undrained shear strength, $s_{\mathrm{u}}$, is observed at $V \gtrsim 10$, whereas a three-fold higher penetration resistance, associated with mobilisation of a drained shear strength, is observed at $V \lesssim 0 \cdot 1$. Partially drained behaviour is observed and hence partially drained soil strength is mobilised between these threshold velocities. This is directly relevant to sustained loading conditions, as the anchor velocity will change over the loading duration (as will be shown later), and hence the mobilised soil strength is expected to depend on $V$.

\section{Consolidation of soil above the anchor during sustained loading}

In normally consolidated clays, sustained loading can cause an increase in soil strength above the anchor due to the dissipation of excess pore pressures. This can be quantified 
from the undrained shear strength ratio of the soil, $s_{\mathrm{u}} / \sigma_{\mathrm{v}}^{\prime}$, where the vertical effective stress, $\sigma_{\mathrm{v}}^{\prime}$, at the top face of the anchor will be higher than the initial (pre-loading) value due to the net increase in bearing pressure applied to the soil during sustained loading.

\section{EXPERIMENTAL ARRANGEMENT \\ Centrifuge tests using PIV}

The centrifuge tests were carried out at an acceleration of $100 \mathrm{~g}$ in the beam centrifuge at the University of Western Australia (UWA) (Randolph et al., 1991). The anchor tests were conducted in a particle image velocimetry (PIV, White et al., 2003) chamber (as illustrated in Fig. 4) that was located at one end of a larger centrifuge strongbox, with a camera and light-emitting diode (LED) lighting located within the empty remainder of the strongbox. The PIV chamber featured transparent poly(methyl methacrylate) (PMMA) panels at either end and was bisected with $25 \mathrm{~mm}$ thick aluminium, allowing for two separate soil samples, each with dimensions $340 \times 100 \times 300 \mathrm{~mm}$ (width $\times$ depth $\times$ height) . The sample width was sufficient to accommodate two anchor tests (each with a plate breadth, $B=25 \mathrm{~mm}$ ) in each sample. The anchor was loaded using an electrical actuator, with the anchor resistance measured using an axial load cell located on the vertical axis of the actuator and the (vertical)

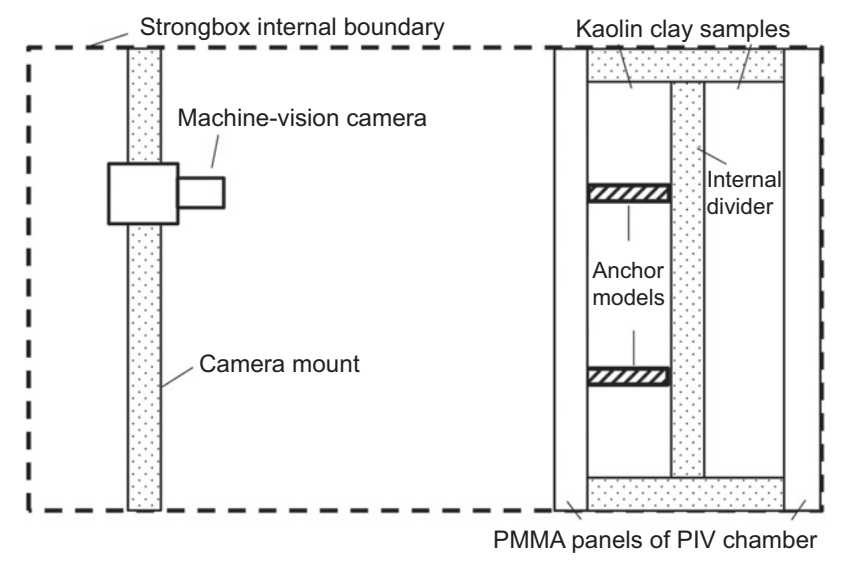

Fig. 4. Experimental arrangement anchor displacement measured using the optical encoder on the actuator motor. Other examples of experimental studies using PIV techniques in the UWA drum centrifuge include Teh et al. (2008), Mana et al. (2012) and Stanier et al. (2014).

\section{Model anchor and soil sample}

The model strip anchor (see Fig. 5(a)) was fabricated from aluminium and had a breadth, $B=25 \mathrm{~mm}$ (which at $100 \mathrm{~g}$ is equivalent to $B=2.5 \mathrm{~m}$ in prototype scale, with a summary of centrifuge scaling laws provided in Garnier et al. (2007)) and a length, $L$, that was identical to the depth of the sample (i.e. $L=100 \mathrm{~mm}$ or $L=10 \mathrm{~m}$ in prototype scale), such that plane strain conditions were achieved. The initial test was conducted using a plate anchor with a thickness, $t=4 \mathrm{~mm}$, although this was increased to $t=5.6 \mathrm{~mm}$ for subsequent tests owing to structural integrity concerns. Each end of the anchor featured slots that accommodated rubber O-rings to maintain the sealed contact between the anchor ends and the PMMA panels. Although the O-rings were greased to minimise friction, analysis of the uplift loads developed during testing indicated large variation in the magnitude of friction mobilised during testing, so a direct comparison between the measured loads in each test was not possible (normalised displacements will be used instead, as will be described in the next section 'Testing procedures and programme').

Normally consolidated kaolin clay samples were prepared by mixing commercially available dry kaolin powder with water under vacuum (close to $-100 \mathrm{kPa}$ ) to form slurry with a moisture content of $120 \%$, corresponding to twice the liquid limit (see Table 1). After mixing for $24 \mathrm{~h}$, the slurry was poured into the PIV chamber and consolidated in-flight at $100 \mathrm{~g}$ for an initial consolidation period of 4 days, with a typical set of testing (in one sample) taking an additional 3 days. Extra slurry was periodically added to the surface of the sample during consolidation to obtain a final sample height of about $215 \mathrm{~mm}$. A $80 \mathrm{~mm}$ layer of free water was maintained above the sample surface to ensure saturation. The initial consolidation time ( $t=4$ days) corresponds with a time factor of $T=c_{\mathrm{v}} t / D^{2} \sim 3$, where $c_{\mathrm{v}}=3.2 \mathrm{~m}^{2} /$ year is the average coefficient of consolidation over the depth of sample and $D=107.5 \mathrm{~mm}$ is the drainage path length (based on a final sample height of $215 \mathrm{~mm}$ with two-way drainage). The

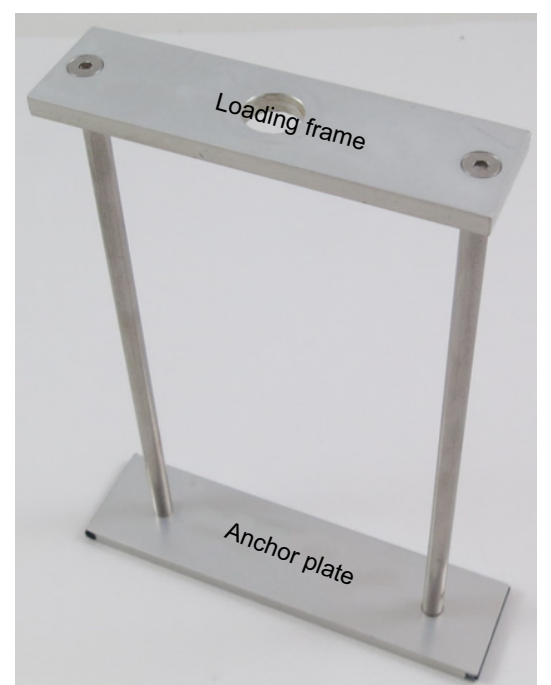

(a)

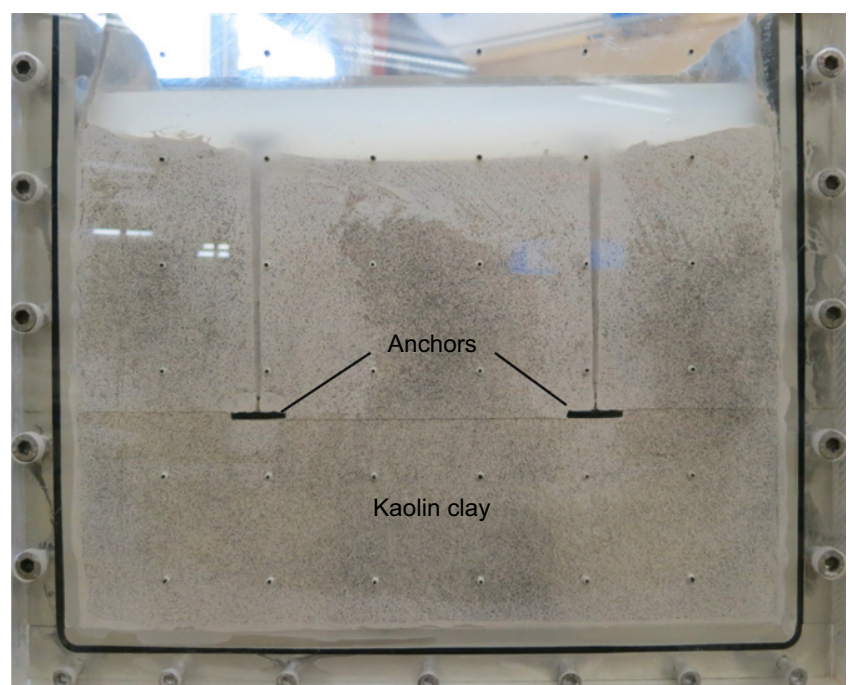

(b)

Fig. 5. Photographs of: (a) model anchor and loading frame; (b) anchors after installation in the kaolin clay 
Table 1. Characteristic parameters of kaolin clay (after Stewart, 1992)

\begin{tabular}{l|c}
\hline Parameter & Value \\
\hline Slope of virgin consolidation line, $\lambda$ & $0 \cdot 205$ \\
Slope of swelling line, $\kappa$ & $0 \cdot 044$ \\
Internal friction angle, $\phi^{\prime}$ & $23^{\circ}$ \\
Poisson ratio, $v$ & $0 \cdot 3$ \\
Void ratio at $p^{\prime}=1 \mathrm{kPa}$ on virgin consolidation line, $e_{\mathrm{N}}$ & $2 \cdot 252$ \\
Liquid limit & $61 \%$ \\
Plastic limit & $27 \%$ \\
\hline
\end{tabular}

theoretical time factor for $99 \%$ consolidation is $T=1 \cdot 78$, which is less than the actual consolidation time allowed, meaning that the sample should have been fully consolidated before the start of the tests.

The undrained shear strength of the sample was determined in-flight by T-bar penetrometer tests (Stewart \& Randolph, 1994) prior to and/or after each model test. The T-bar probe had a diameter $d=5 \mathrm{~mm}$ and was $20 \mathrm{~mm}$ long, and was penetrated at a constant velocity, $v=1 \mathrm{~mm} / \mathrm{s}$, such that the dimensionless velocity, $v d / c_{\mathrm{v}}$ was in the range $35-158$ (where the coefficient of consolidation, $c_{\mathrm{v}}=1-4.5 \mathrm{~m}^{2} /$ year over the T-bar penetration depth) thus ensuring undrained conditions (House et al., 2001). The undrained shear strength, $s_{\mathrm{u}}$, of the samples was determined from the T-bar penetration resistance using the commonly adopted T-bar bearing factor of $10 \cdot 5$. As expected for a normally consolidated soil, the strength varied linearly with depth, with an average gradient in the range $0.94-1.41 \mathrm{kPa} / \mathrm{m}$ (in prototype scale), depending on the elapsed time between sample preparation and the T-bar tests. The average effective unit weight was $6.2 \mathrm{kN} / \mathrm{m}^{3}$, as inferred from moisture content measurements made on a core taken from the sample after testing. The coefficient of vertical consolidation, $c_{\mathrm{v}}$, was quantified through one-dimensional consolidation tests reported by Richardson et al. (2009): $c_{\mathrm{v}}$ increases with vertical effective stress, $\sigma_{\mathrm{v}}^{\prime}$, from $c_{\mathrm{v}}=1.4 \mathrm{~m}^{2} /$ year near the soil surface $\left(\sigma_{\mathrm{v}}^{\prime}=20 \mathrm{kPa}\right)$ to $c_{\mathrm{v}}=3.3 \mathrm{~m}^{2} /$ year at the average initial anchor embedment depth of $z_{\mathrm{i}}=4 \cdot 6 B\left(\sigma_{\mathrm{v}}^{\prime}=71 \mathrm{kPa}\right)$.

\section{Testing procedures and programme}

After consolidation, the centrifuge was spun down and the PIV chamber was removed from the centrifuge strongbox. The PMMA panels of the chamber were removed and the model anchors were inserted into the clay using a custom fabricated guide that ensured the plate remained horizontal. The models were embedded at a normalised depth $z_{\mathrm{i}} / B$ between 4.51 and $4 \cdot 78$, which is sufficient to mobilise a deep flow failure mechanism (Song et al., 2008; Wang et al., 2010). It is noted that in SEPLA field applications, anchor plates are inserted vertically to the targeted embedment depth, followed by a rotational keying process. However, the tests performed in this study simplified this installation process by conducting horizontal insertion from the side of the sample, in order to minimise the influence of excess pore pressures and stress field disturbance that otherwise would have occurred had the models been installed vertically (i.e. from the sample surface) and subsequently keyed to a horizontal orientation. Black flock powder was passed through a $300 \mu \mathrm{m}$ sieve and subsequently scattered evenly over the exposed clay surface to add the spatial variability in contrast necessary for the PIV measurements. The optimal flock density was based on the calibration technique proposed by Stanier \& White (2013). The PMMA panel was then reattached to the PIV chamber and the chamber
Table 2. Centrifuge and numerical testing programme

\begin{tabular}{l|c|c}
\hline \multirow{2}{*}{ PIV test } & Initial embedment & Targeted displacement \\
\cline { 2 - 3 } & $z_{\mathrm{i}} / B$ & $w_{\mathrm{p}} / w_{\mathrm{f}}$ \\
\hline PIV-MP & $4 \cdot 72$ & 0 \\
PIV-CW11 & $4 \cdot 63$ & $0 \cdot 11$ \\
PIV-CW19 & $4 \cdot 67$ & $0 \cdot 19$ \\
PIV-CW22 & $4 \cdot 78$ & $0 \cdot 22$ \\
PIV-CW39 & $4 \cdot 77$ & $0 \cdot 39$ \\
PIV-CW45 & $4 \cdot 59$ & $0 \cdot 45$ \\
PIV-CW55 & $4 \cdot 51$ & $0 \cdot 55$ \\
LDFE analysis & & Targeted load \\
& & $F_{\text {sus }} / F_{\text {max }}$ \\
\hline FE-MP & & 1 \\
FE-CF30 & $4 \cdot 72$ & $0 \cdot 30$ \\
FE-CF45 & $4 \cdot 72$ & $0 \cdot 45$ \\
FE-CF60 & $4 \cdot 72$ & $0 \cdot 60$ \\
FE-CF70 & $4 \cdot 72$ & $0 \cdot 70$ \\
FE-CF84 & $4 \cdot 72$ & $0 \cdot 84$ \\
\hline
\end{tabular}

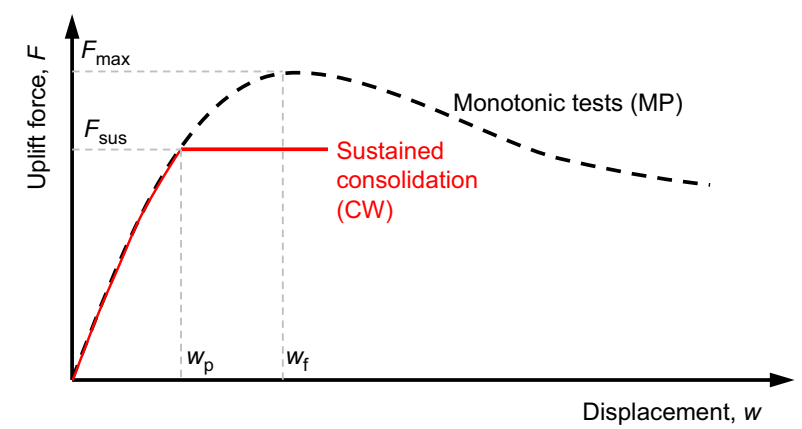

Fig. 6. Illustration of loading steps for monotonic and sustained loading tests

was placed back into the centrifuge strongbox for a reconsolidation period equal to that spent at $1 \boldsymbol{g}$ (typically $1 \mathrm{~h})$ before the anchor tests. Fig. 5(b) shows the anchor models in the PIV chamber prior to testing. Loading of the anchor models was performed subsequently, accompanied by T-bar tests for strength determination immediately before or immediately after the testing. The time between a T-bar test and an anchor test was typically $<10 \mathrm{~min}$, which is negligible compared to the reconsolidation time ( $1 \mathrm{~h})$.

Seven vertically loaded anchor tests were performed, as listed in Table 2. The loading sequence is schematically illustrated in Fig. 6. In the first test (PIV-MP) the anchor was pulled vertically under displacement control until failure was reached, defined as the maximum measured anchor resistance. The anchor velocity was $v=0.3 \mathrm{~mm} / \mathrm{s}$, which corresponds with $V=79$, such that undrained conditions are to be expected (see Fig. 3). The displacement at failure, $w_{\mathrm{f}}$, defined as the anchor displacement when the maximum anchor force $\left(F_{\max }\right)$ was mobilised, served as a reference for the subsequent tests under sustained loading. In the sustained loading tests, the anchor was initially loaded under displacement control at $v=0.3 \mathrm{~mm} / \mathrm{s}$, until a targeted displacement, $w_{\mathrm{p}}$, was reached, as indicated in Table 2 and shown in Fig. 6. The load achieved at the displacement $w_{\mathrm{p}}, F_{\text {sus }}$, was maintained by immediately switching the actuator from displacement control to load control. $F_{\text {sus }}$ was maintained until either anchor failure occurred (as evident from uncontrolled anchor 
displacement) or when the anchor stabilised at a velocity two orders of magnitude lower than at the beginning of the sustained loading process, indicating that consolidation was nearly achieved.

The test nomenclature is based on the type of loading (PIV-MP for monotonic uplift, PIV-CW for consolidated sustained loading) and the targeted normalised displacement $w_{\mathrm{p}} / w_{\mathrm{f}}$. Owing to the variable friction at the anchor-PMMA interface, the measured anchor loads are not considered to be representative of the load associated solely with geotechnical shear resistance. Therefore, the sustained loading tests were based on the targeted normalised displacement, $w_{\mathrm{p}} / w_{\mathrm{f}}$, rather than the normalised force, $F_{\text {sus }} / F_{\max }$, based on the assumption that the relationship between $w_{\mathrm{p}} / w_{\mathrm{f}}$ and $F_{\text {sus }} / F_{\max }$ is unique in a given test.

\section{EXPERIMENTAL RESULTS}

Monotonic failure mechanism

The maximum resistance in the monotonic loading test was measured at a displacement ratio, $w_{\mathrm{f}} / B=0 \cdot 18$. A PIV analysis was conducted on the six consecutive images captured every $1 / 3 \mathrm{~s}$ immediately after the ultimate capacity. The results of this analysis are presented as normalised vectorial displacement fields on the left-hand side of Fig. 7, where the vectors represent instantaneous movements of the soil surrounding the anchor immediately after the maximum resistance was reached, and are normalised by the anchor velocity. The same measurements are presented as contours of normalised velocity on the right-hand side of Fig. 7. At the base of the anchor, the failure mechanism resembles the full flow-round mechanism that is typically associated with the behaviour of deeply buried plate anchors (Martin \& Randolph, 2001). However, a dissymmetry of the flow-round mechanism is evident at the top of the anchor, which becomes more pronounced close to the centre of the plate (i.e. at $x / B=0$, where $x$ is the horizontal distance from the plate centre). This is different from the symmetrical full-flow mechanism that is typically observed, and is potentially due to the rod on the loading frame (see Fig. 5), which was located $0 \cdot 6 B$ from the anchor end, and may have caused soil movements to be restrained in a region between the rod and the PMMA panel.

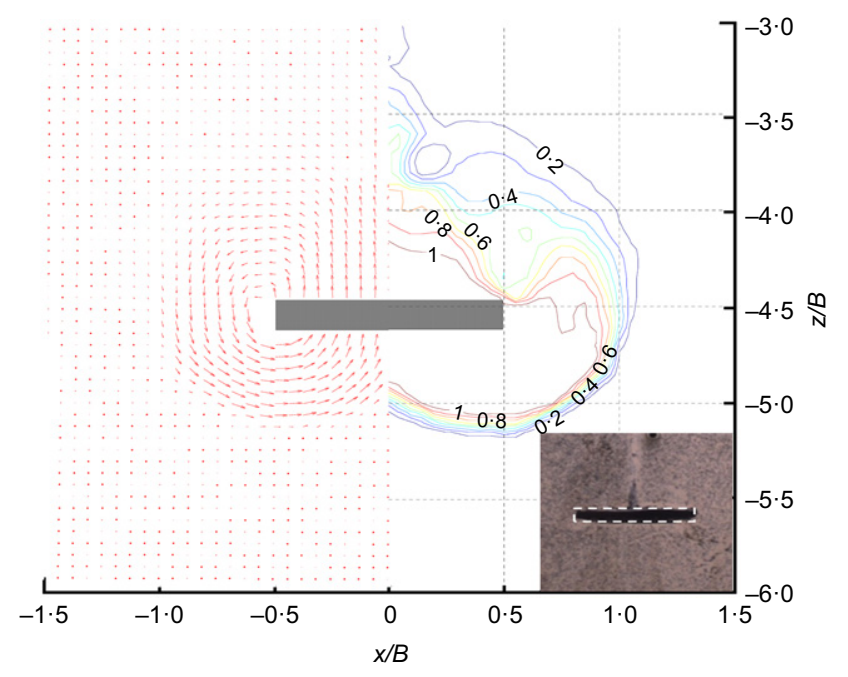

Fig. 7. Failure mechanism in the test PIV-MP (left: incremental displacement vectors; right: contours of normalised incremental displacements
Sustained loading

Anchor displacement. In the sustained loading tests, the anchor was loaded under displacement control at $0.3 \mathrm{~mm} / \mathrm{s}$ until the target displacement was reached, at which point the measured resistance (which comprises the net pulling force plus the friction developed at the anchor-PMMA interface), was maintained for consolidation to take place. Fig. 8 presents the development of anchor displacement, $w_{\mathrm{s}}$, with nondimensional consolidation time, $T=c_{\mathrm{v}} t_{\mathrm{s}} / B^{2}$, where $t_{\mathrm{s}}$ is the time during sustained loading, for all the sustained loading tests. The value of $c_{\mathrm{v}}$ was taken as $3.3 \mathrm{~m}^{2} /$ year, as appropriate for the vertical effective stress at the initial embedment depth $(\sim 4 \cdot 6 B)$. Fig. 8 shows that unstable conditions occurred for $w_{\mathrm{p}} / w_{\mathrm{f}} \geq 0.45$, characterised by continuously increasing displacements until the load cannot be sustained any longer, whereas stable conditions occurred for $w_{\mathrm{p}} / w_{\mathrm{f}} \leq 0 \cdot 39$, in which anchor displacement stabilises eventually. Fig. 8 also shows the expected increase in the magnitude of displacement at the end of consolidation with the level of sustained load.

Accordingly, the threshold of the net force that can be sustained indefinitely corresponds to a normalised anchor displacement, $w_{\mathrm{p}} / w_{\mathrm{f}}$, that lies in the range $0 \cdot 39-0 \cdot 45$. Further insights into the anchor behaviour during consolidation at different sustained load levels are presented in Fig. 9, which shows the non-dimensional anchor velocity with nondimensional consolidation time.

In the tests that failed during sustained loading $\left(w_{\mathrm{p}} / w_{\mathrm{f}}=0.45\right.$ and $\left.0 \cdot 55\right)$, the anchor velocities were generally sufficient to eventually generate an undrained response $(V \gtrsim 10)$ and increased rapidly at the onset of failure (at $T \sim 0.6$ and 0.02 for $w_{\mathrm{p}} / w_{\mathrm{f}}=0.45$ and 0.55 , respectively). Among the four tests below the threshold displacement level, two distinct behaviours can be identified. For the three lowest levels of sustained loading $\left(w_{\mathrm{p}} / w_{\mathrm{f}}=0 \cdot 11,0 \cdot 19\right.$ and $\left.0 \cdot 22\right)$, the

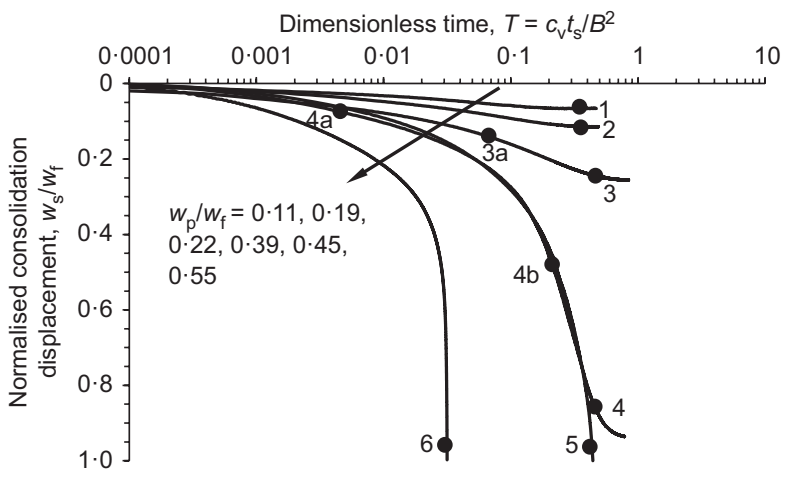

(a)

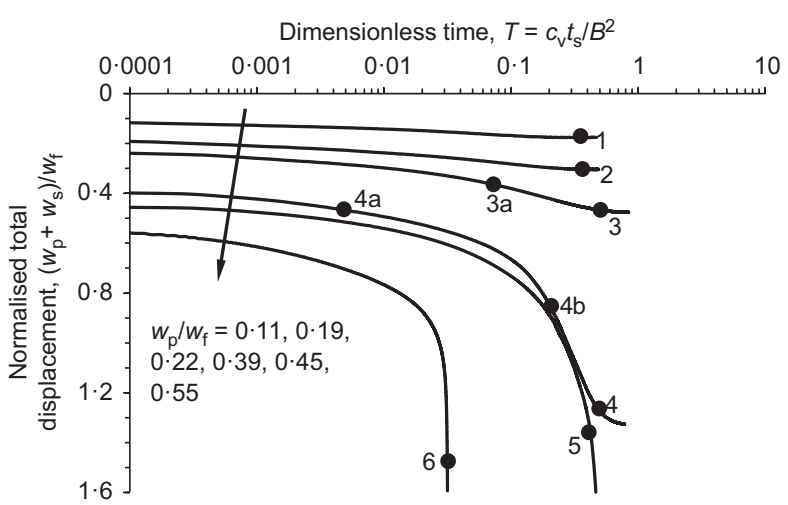

(b)

Fig. 8. Anchor displacement during centrifuge sustained loading tests: (a) relative displacement; (b) total displacement 


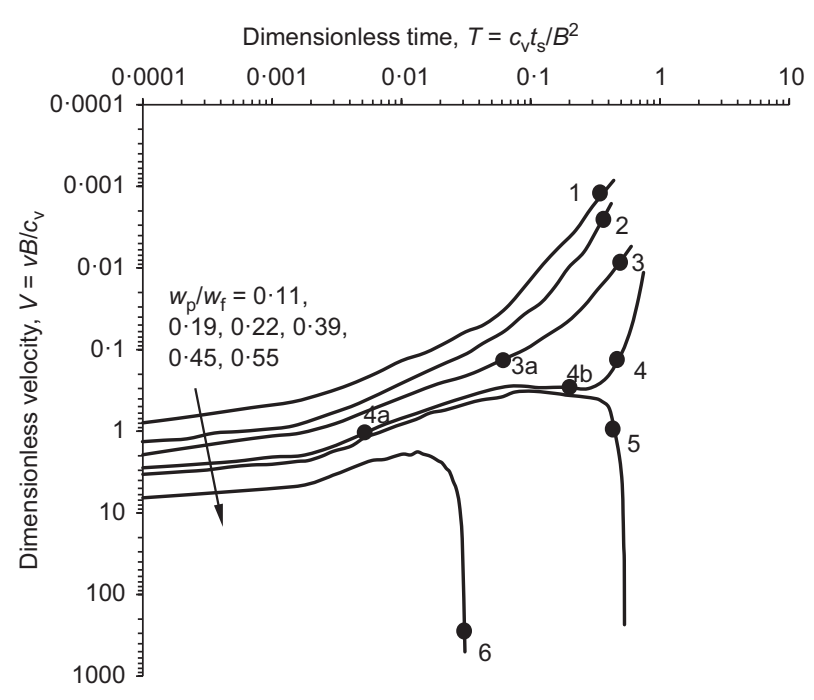

Fig. 9. Dimensionless anchor velocity during centrifuge sustained loading tests

anchor velocity reduced continuously during consolidation from initially partially drained conditions $(V=0 \cdot 8-2)$ to drained conditions $(V=0 \cdot 1)$ at between $T=0 \cdot 02$ and $T=0 \cdot 1$. In contrast, for the test with the highest (stable) sustained loading level $\left(w_{\mathrm{p}} / w_{\mathrm{f}}=0 \cdot 39\right)$, the anchor velocity initially followed the response for $w_{\mathrm{p}} / w_{\mathrm{f}}=0 \cdot 45$, which eventually failed, but at $T \sim 0 \cdot 4$ (and when partially drained conditions were being generated; $V=0 \cdot 3$ ) the velocity suddenly reduced. The reasons for this metastable condition and for the anchor velocity to reduce abruptly are related to the behaviour of the soil at the base of the anchor, as will be revealed by the PIV measurements later.

PIV analysis. Figure 10 shows the soil flow mechanism at instants when the degree of consolidation (defined as the ratio of current to total displacement during sustained loading) $U=90 \%$ for stable tests (corresponding to points 1-4 in Fig. 8) and when failure has occurred for tests that failed during sustained loading (corresponding to points 5 and 6 in Fig. 8). The left-hand sides in Fig. 10 illustrate the incremental soil displacement vectors over a time interval corresponding to the last $0 \cdot 005 \mathrm{~B}$ of anchor displacement, normalised by the anchor displacement $(0.005 B)$ within the same time interval. The right-hand sides in Fig. 10 show contours of the normalised incremental soil displacement (or normalised velocity). Images of the soil surrounding the plate for each sustained loading test taken at points 1-6 in Fig. 8 are shown as insets in Fig. 10, recalling that the anchor thickness for the first test was $4 \mathrm{~mm}$ compared with $5.6 \mathrm{~mm}$ for all subsequent tests. Motivated by the PIV observations at $U=90 \%$ for tests PIV-CW22 $\left(w_{\mathrm{p}} / w_{\mathrm{f}}=0 \cdot 22\right)$ and PIV-CW39 $\left(w_{\mathrm{p}} / w_{\mathrm{f}}=0 \cdot 39\right)$, additional PIV analyses were conducted at $U=50 \%$ for test PIV-CW22, and at $U=10$ and $50 \%$ for test PIV-CW39. The results from these additional analyses are provided in Fig. 11 using the same presentation format as in Fig. 10. Observations from these PIV mechanisms, and recalling the drainage conditions identified during each test in Fig. 9, lead to the following comments.

(a) In the tests with $w_{\mathrm{p}} / w_{\mathrm{f}}=0.45$ and 0.55 , both of which failed during sustained loading, the soil failure mechanism (points 5 and 6 on Fig. 10) resembles the flow-round mechanism observed for the monotonic test in Fig. 7. The volume of mobilised soil involved in the mechanism appears to increase with $V$ (comparing point $5, w_{\mathrm{p}} / w_{\mathrm{f}}=0 \cdot 45, V=1$ with point $6, w_{\mathrm{p}} / w_{\mathrm{f}}=0 \cdot 55$, $V=379$ in Fig. 10). As the dimensionless velocity, $V=379$ for point 6 is higher than that required for undrained conditions, suction is expected to be generated at the base of the anchor such that the soil remains bonded to the base of the plate. This is supported by the PIV measurements at point 6 (see Fig. 10), which show essentially identical anchor velocities immediately above and below the plate. At point $5\left(w_{\mathrm{p}} / w_{\mathrm{f}}=0.45\right)$ the dimensionless velocity, $V=1$, which is expected to generate partially drained conditions. This is still sufficient for generation of suction (albeit of lower magnitude than at point 6 with $V=379$ ), such that formation of a gap due to the absence of suction is not expected, and indeed is not observed (Fig. 10).

(b) For stable tests in which the lowest loads $\left(w_{\mathrm{p}} / w_{\mathrm{f}}=0 \cdot 11\right.$ and $0 \cdot 19)$ were sustained indefinitely, similar patterns of soil flow are shown at $90 \%$ consolidation (points 1 and 2). In contrast to the full-flow mechanism shown in Fig. 7, the mechanisms are characterised by pure vertical displacements within a smaller region above and beneath the anchor. The normalised velocities adjacent to both the top and base of the plate are equal to unity, inferring that the soil is fully bonded to the anchor, which is also evident from the corresponding images for points 1 and 2 .

(c) For the tests with $w_{\mathrm{p}} / w_{\mathrm{f}}=0 \cdot 22$ and $0 \cdot 39$, the patterns of soil deformation at $90 \%$ consolidation (points 3 and 4 ) differ from those at points 1 and 2, even though all relate to stable anchor behaviour. Fig. 10 shows a lower normalised velocity at the base of the plate than above the plate for point 3 , which indicates the onset of gap formation. This is more evident for point 4 as both the PIV and the image of the anchor-soil interface clearly show breakaway at the base of the anchor. Further PIV analyses for test PIV-CW22 $\left(w_{\mathrm{p}} / w_{\mathrm{f}}=0 \cdot 22\right)$ at $U=50 \%$ (point 3a in Fig. 8) are shown in Fig. 11, where it is evident that the mechanism is similar to that observed at points 1 and 2; that is, the soil movement is purely vertical above and under the plate and the soil remains fully bonded to the base of the plate. As noted above, this transitions at $U=90 \%$ (point 3 in Fig. 10), at which point soil displacements beneath the anchor become localised and are about half of those above the anchor as the gap begins to form. However, this point coincides with completion of the anchor displacement, such that the emergence of the gap was somewhat subtle for this example.

(d) At higher loads (corresponding with $w_{\mathrm{p}} / w_{\mathrm{f}}=0 \cdot 39$ ), the overall pattern of soil displacement at $U=10 \%$ is similar to that at points 1,2 and $3 \mathrm{a}$, although involving a larger volume of soil due to the higher anchor velocity $(V=1 \cdot 2$ compared with $V \leq 0 \cdot 15$ ). At $U=50 \%$, a wedge of soil above the plate moves upwards at a similar velocity to the anchor, and soil at the top edge of the plate flows to the base of the plate. However, the flow around the plate sides does not fully extend to the middle of the plate base (i.e. the soil immediately underneath the anchor moves slower than the plate), which indicates initiation of breakaway (also shown in the inset image on Fig. 11 for point 4b). At $U=90 \%$, the anchor velocity is reduced to $V=0.15$ from $V=0.31$ at $U=50 \%$, and hence the mobilised volume of soil at the anchor base is reduced. It is evident that at $U=90 \%$ there is growing dissymmetry of soil displacement between the top and base of the plate, and the displacement vectors beneath the base are primarily 

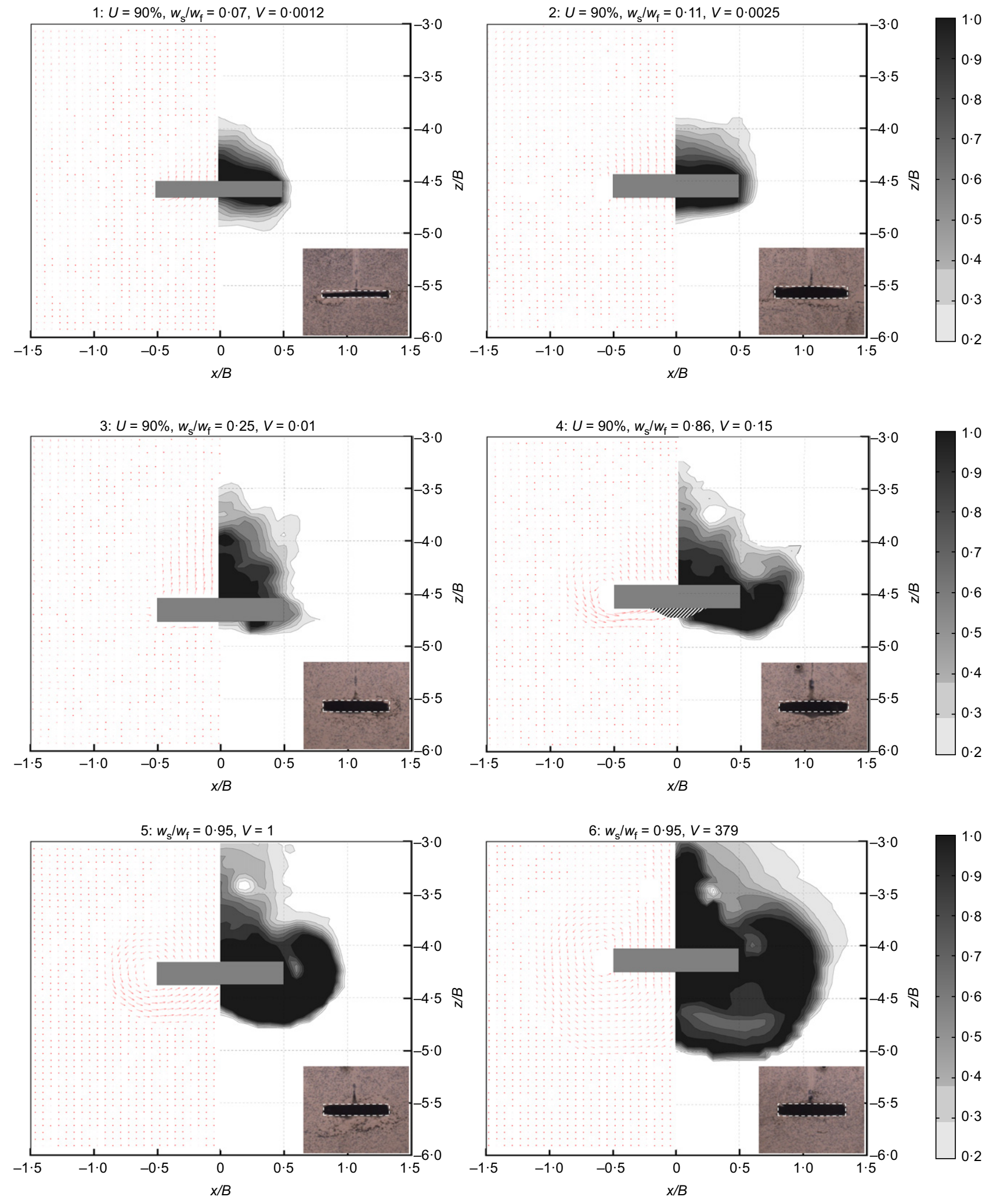

Fig. 10. Soil deformation mechanisms at $U=90 \%$ for stable tests (points 1-4) and when failure has occurred for failed tests (points 5 and 6 ) (left side of diagram: incremental displacement vectors; right side of diagram: contours of normalised incremental displacements)

horizontal, meaning that the soil backflow under the plate does not evolve to soil uplift.

(e) An interesting observation is the stability of the anchor even after breakaway. This is considered to be due to the dissipation of excess pore pressures that occurred prior to breakaway, which led to a consolidation-induced soil strength increase that was sufficient to compensate for the reduction in potential capacity due to breakaway.
In summary, consideration of the anchor velocity, degree of consolidation and observation from the PIV analyses suggests that the stability of plate anchors under sustained loading is controlled by: $(a)$ the dimensionless velocity of the anchor, as this will inform the drainage conditions generated and hence the soil strength mobilised by the anchor; $(b)$ the extent to which excess pore pressures at the top of the anchor dissipate and lead to consolidation-induced strength 

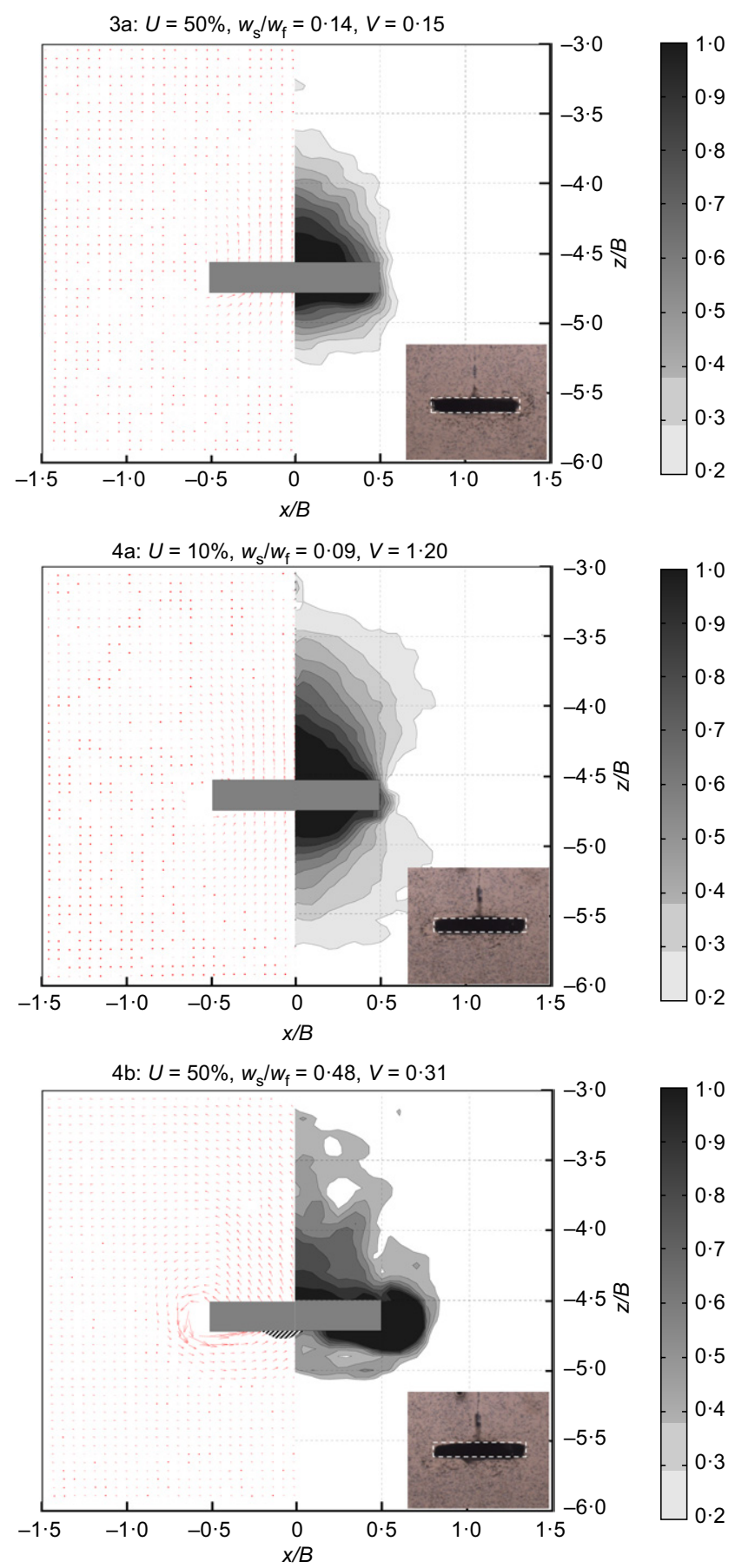

Fig. 11. Soil deformation mechanisms at $U=50 \%$ (point 3a) in test PIV-CW22 and $U=10 \%$ (point $4 a$ ) and $50 \%$ (point $4 \mathrm{~b}$ ) in test PIV-CW39 (left side of diagram: incremental displacement vectors; right side of diagram: contours of normalised incremental displacements)

increases; and (c) the potential for a gap to form at the base of the plate. Overall stability is governed by a combination of these factors. For instance, and as demonstrated here through test PIV-CW39 $\left(w_{\mathrm{p}} / w_{\mathrm{f}}=0.39\right)$, the reduction in capacity that may occur due to breakaway is offset by a combination of strength increases due to the dissipation of excess pore pressures and the associated higher mobilised strengths due to the prevalence of drained, or near-drained conditions.

Breakaway interpretation. The variation in soil flow and anchor behaviour throughout consolidation may be

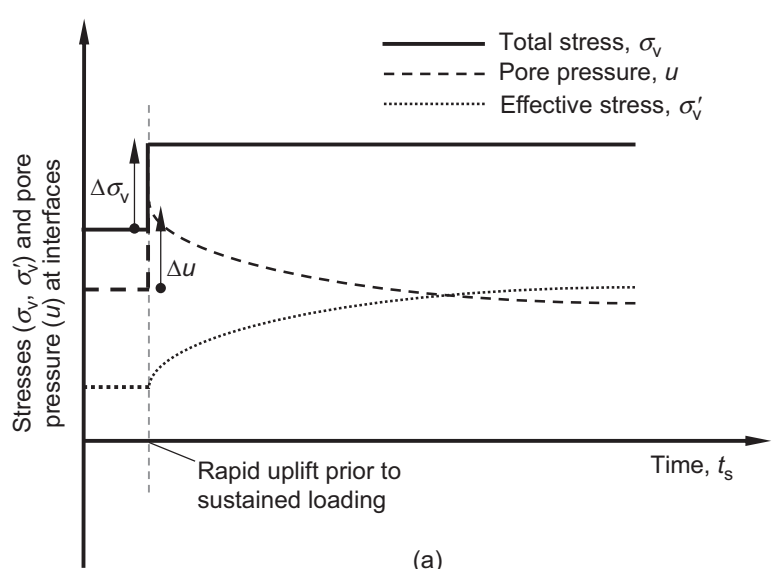

(a)

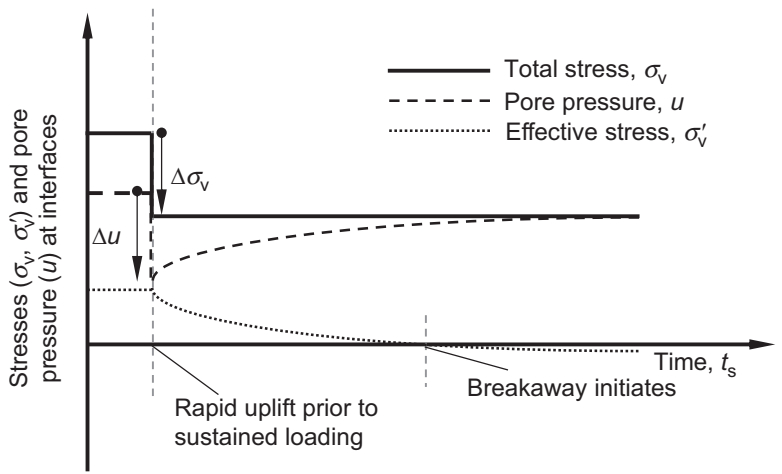

(b)

Fig. 12. Schematic illustration of changes in stresses $\left(\sigma_{\mathrm{v}}\right.$ and $\left.\sigma_{\mathrm{v}}^{\prime}\right)$ and dissipation of pore pressures $(u)$ throughout sustained loading test at (a) top and (b) bottom interfaces

interpreted in terms of changes in total stresses, $\sigma_{\mathrm{v}}$, dissipation of excess pore pressures, $u$, and hence changes in effective stresses, $\sigma_{\mathrm{v}}^{\prime}$, as illustrated in Fig. 12 (noting that this is a schematic interpretation, as no pressures were measured in the test). During the initial loading, undrained uplift induces an increase in total stresses and corresponding positive excess pore pressures at the top of the anchor, while total stresses at the base of the anchor are reduced and hence negative excess pore pressures (i.e. suctions) are generated. The magnitude of the excess pore pressure generated is directly proportional to the magnitude of the total stress applied (i.e. to the magnitude of the sustained load). For lower levels of sustained load the reduction in total stresses at the base of the anchor (and the corresponding suction generated) is low relative to the geostatic effective stresses, and the effective stress at the base of the anchor remains positive throughout consolidation. Therefore, the soil remains fully bonded to the base of the anchor. This is the case for tests at low values of sustained load, specifically for $w_{\mathrm{p}} / w_{\mathrm{f}}=0 \cdot 11$ and $0 \cdot 19$, in which a localised mechanism characterised by vertical displacements above and below the plate (see points 1 and 2 in Fig. 10) is observed.

If the change in total stress induced by initial loading is significant relative to the geostatic effective stress, the vertical effective stress at the base of the anchor will become nil at some point during consolidation, initiating breakaway, as is the case for the test with $w_{\mathrm{p}} / w_{\mathrm{f}}=0 \cdot 39$. However, in this case the breakaway, which should mobilise a reduced capacity factor, did not lead to anchor failure. There are two reasons for this. First, the anchor normalised velocity at the onset of breakaway is $0 \cdot 31$, such that the response is either partially drained or drained and hence the mobilised strength is higher 
than would otherwise be mobilised under undrained conditions. Second, the soil strength at the top of the anchor is higher owing to the increase in effective stresses during consolidation.

For very high changes in total stresses, induced by higher levels of sustained load such as the tests with $w_{\mathrm{p}} / w_{\mathrm{f}}=0.45$ and 0.55 , breakaway would be expected to occur as the effective stresses at the base of the anchor are reduced to zero. However, in these particular cases, the anchor experiences continuous displacement at velocity $(V \gtrsim 10)$, close to the undrained range, resulting in: $(a)$ limited strength enhancement due to consolidation at the top of the anchor and $(b)$ re-mobilisation of negative excess pore pressures at the base of the anchor, preventing the development of a gap. As the soil strength increases with depth, further migration of the anchor into new soil results in an increase of the anchor velocity until the anchor is completely pulled out. This is in contrast with tests at $w_{\mathrm{p}} / w_{\mathrm{f}}=0 \cdot 39$, which experienced similar total displacements to those of tests at $w_{\mathrm{p}} / w_{\mathrm{f}}=0.45$ (see Fig. 8(b)), but a lower level of sustained loading. The interpretation above is further investigated and validated in the following numerical analyses.

\section{FINITE-ELEMENT ANALYSIS \\ Analysis method}

It is worth reiterating that the PIV centrifuge tests described here do not accurately quantify the uplift resistance of the anchor owing to the friction that developed along the PMMA window. In order to quantify the threshold sustained load that can be applied without leading to failure, and to numerically demonstrate the hypothesis outlined in the previous section, two-dimensional large-deformation finiteelement (LDFE) analyses were conducted using the remeshing and interpolation technique with small strain (RITSS) approach incorporating the modified Cam-Clay (MCC) model. The RITSS strategy was proposed by $\mathrm{Hu}$ \& Randolph (1998) and then coupled with the commercial FE package, Abaqus, as described in Wang et al. (2010, 2011, 2013). In the RITSS analysis the rapid uplift of the plate anchor was divided into typically dozens of small steps. In each step, the pulling distance was small enough to avoid severe mesh distortion around the anchor. The deformed soil was then remeshed and an updated Lagrangian calculation was performed, followed by mapping of the field variables (e.g. effective stresses, pore water pressures and material properties) to the new mesh at the commencement of the next step. The detailed procedure of Abaqus-based RITSS can be found in Wang et al. (2010, 2015).

The soil was discretised with quadratic quadrilateral elements with reduced integration (CPE8RP in Abaqus). The pore pressure was only calculated at four corner nodes of the element. Owing to geometrical symmetry, only half of the anchor and soil were modelled. The horizontal and vertical extents of the soil mesh were $6 B$ and $10 \cdot 72 B$, respectively, which is sufficient to eliminate boundary effects, as confirmed through initial numerical trials with varying horizontal and vertical extents. Drainage was allowed at the top and bottom surfaces of the soil domain to simulate the two-way drainage in the centrifuge sample.

The material properties assumed in the MCC model are listed in Table 1 . The normally consolidated clay was initially set at a geostatic state with a coefficient of earth pressure, $K_{0}=1-\sin \phi^{\prime}$. The undrained shear strength was inferred from the MCC model (Wroth, 1984) and increased with the effective mean stress as

$$
s_{\mathrm{u}}=\frac{2}{\sqrt{3}} \frac{M p^{\prime}}{2^{1+\Lambda}}\left[\left(\frac{q}{M p^{\prime}}\right)^{2}+1\right]^{\Lambda} \text { with } M=\frac{6 \sin \phi^{\prime}}{3-\sin \phi^{\prime}}
$$

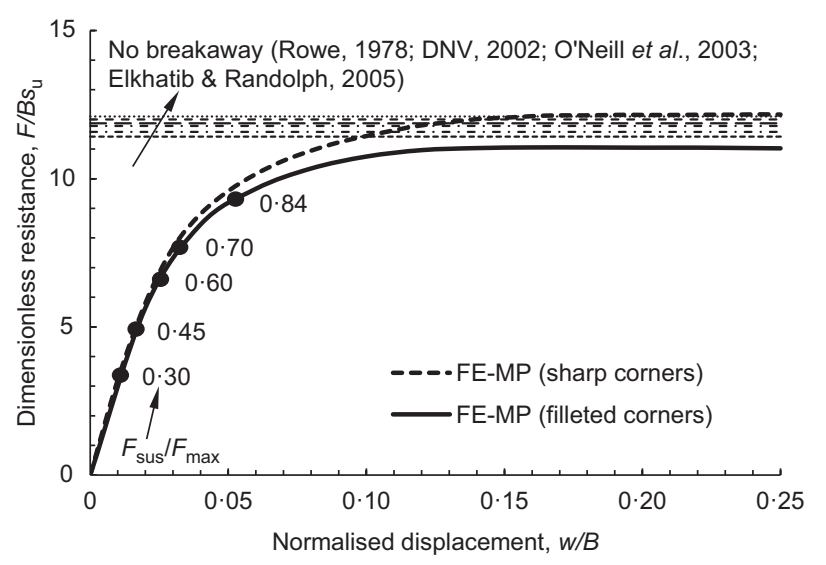

Fig. 13. Dimensionless force plotted against displacement curves under monotonic uplift from FE analyses (the closed circles represent values of $F_{\text {sus }} I F_{\max }$ from subsequent sustained loading analyses)

where $\Lambda=(\lambda-\kappa) / \lambda$, and $M$ is the slope of critical state line in $q-p^{\prime}$ space, derived from triaxial compression tests. Therefore, the $s_{\mathrm{u}}$ profile increased linearly with depth with a gradient equal to $1.73 \mathrm{kPa} / \mathrm{m}$. Although this is approximately $23 \%$ higher than the upper bound gradient observed in the centrifuge tests, the absolute magnitude does not affect the qualitative comparison presented hereafter.

As suggested by Mahmoodzadeh et al. (2014, 2015), the permeability of kaolin clay may be expressed as

$$
\begin{aligned}
k= & 1.06 \times 10^{-8} \frac{\gamma_{\mathrm{w}} \lambda\left(1+2 K_{0}\right)}{(1+e) \exp \left[\left(e_{\mathrm{N}}-C-e\right) / \lambda\right]} \\
& \times \sqrt{1+\frac{0.42 \exp \left[\left(e_{\mathrm{N}}-C-e\right) / \lambda\right]}{1+2 K_{0}}} \mathrm{~m} / \mathrm{s}
\end{aligned}
$$

where the not-yet-defined parameters are the void ratio, $e$, the unit weight of water, $\gamma_{w}=9.8 \mathrm{kN} / \mathrm{m}^{3}$, and the distance between the virgin consolidation line and the normal consolidation line, $C=0.048$ (from the parameters in Table 1).

The anchor was modelled as a $0 \cdot 22 B$ thick porous rigid body, with a permeability that was four orders lower than that of the soil. The anchor surfaces were assumed to be smooth, given that the roughness of an anchor-soil interface has marginal effect on the undrained capacity (Rowe \& Davis, 1982; Wang et al., 2010). Separation between the anchor base and soil was allowed (but not imposed) when $\sigma_{\mathrm{v}}^{\prime}$ was less than zero. As the anchor was deeply embedded, if a gap formed, it could only be filled by seepage of pore water from the surrounding soil. Pore water was allowed to seep into the gap formed at the anchor base if breakaway occurred under sustained loading. This was achieved numerically by maintaining continuity of excess pore pressures on both sides of contact surfaces when separation occurs. Before loading, the anchor was wished-in-place at an embedment depth of $4 \cdot 72 \mathrm{~B}$, consistent with the installation approach adopted in the centrifuge tests.

\section{Monotonic loading}

In the monotonic loading analysis (FE-MP in Table 2), the anchor was displaced at $V=79$, identical to that in the corresponding centrifuge test. The resulting dimensionless load-normalised displacement relationship is shown in Fig. 13, and shows a limiting capacity factor, $N_{\mathrm{c}}=12 \cdot 14$ at an anchor displacement, $w_{\mathrm{f}}=0 \cdot 16 \mathrm{~B}$ for an anchor with sharp corners (shown by the dashed line in Fig. 13). This value is slightly higher than the upper bound solutions, $N_{\mathrm{c}}=11.42$ and $12 \cdot 1$, for a vanishingly thin plate, deduced by Rowe 


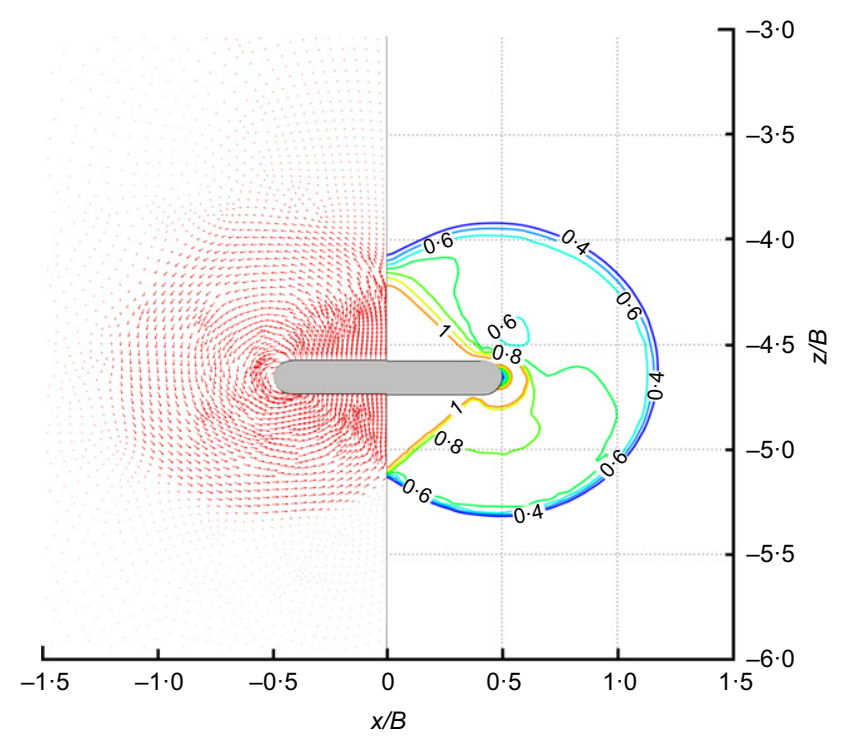

Fig. 14. Failure mechanism in the test FE-MP (left: incremental displacement vectors; right: contours of normalised incremental displacements)

(1978) and O'Neill et al. (2003), respectively, and is attributed to a combination of the higher plate thickness and mesh density (as also demonstrated in Wang \& O'Loughlin (2014)). To avoid severe stress concentrations and local mesh distortions that are detrimental to numerical stability (particularly in the subsequent sustained loading step), the side edges of the plate were filleted as semicircles. The loaddisplacement curve for an anchor with rounded edges is also presented in Fig. 13, which predicts a slightly lower $N_{\mathrm{c}}=11.05$ at $w_{\mathrm{f}}=0.14 \mathrm{~B}$. This limiting value from the analysis of the filleted anchor will be used in the following sections as the reference case for the sustained loading tests.

During the PIV tests, the anchor failed during consolidation if it was subjected to a sustained load at a displacement $w_{\mathrm{p}}=0.45 w_{\mathrm{f}}$ prior to consolidation. Assuming the same threshold $\left(w_{\mathrm{p}} / w_{\mathrm{f}}=0.45\right)$ applies in the LDFE analyses, the numerically determined $w_{\mathrm{f}}=0 \cdot 14 B$ at the limiting $N_{\mathrm{c}}$ corresponds to $w_{\mathrm{p}}=0 \cdot 06 \mathrm{~B}$. From Fig. 13 the corresponding threshold load (at $w=0 \cdot 06 B$ ) from the LDFE results is inferred as $F_{\text {sus }} / F_{\max }=0.88$, which is reasonably consistent with the experimental threshold ratio of 0.85 reported by Wong et al. (2012).

The failure mechanism (from the LDFE monotonic uplift test FE-MP) immediately after the peak load was reached (at $w_{\mathrm{f}} / B=0 \cdot 14$ ) is shown in Fig. 14. The presentation format is the same as that used in Fig. 7, with displacement vectors on the left and contours of soil displacement (normalised by the anchor displacement) on the right. Comparing Fig. 14 with Fig. 7, it is apparent that the numerically and experimentally observed failure mechanisms for monotonic loading are in quite good agreement, particularly in view of the effects caused by the loading frame described previously. Fig. 14 also shows that the soil remains fully attached to the anchor base, as also observed in the monotonic PIV test.

\section{Sustained loading}

To further understand breakaway under sustained loading, five sustained loading LDFE simulations (see Table 2) were carried out with $F_{\text {sus }} / F_{\max }$ in the range $0 \cdot 3-0 \cdot 84$ below the threshold level of around $0 \cdot 88$ identified previously. The five $F_{\text {sus }} / F_{\max }$ ratios are shown by the markers on the load-displacement curve in Fig. 13, which represent the load-displacement state at the start of the subsequent sustained loading stage. As the robustness and accuracy of calculation at contact surfaces is sensitive to the remeshing and mapping procedure, particularly where potential breakaway would occur, consolidation under sustained load was therefore modelled in a single step (i.e. no remeshing) to show clearly the configuration of soilanchor boundaries. The calculation was stopped either when full consolidation was achieved (under low sustained load) or when severe mesh distortion occurred after breakaway (under intermediate sustained load). Note that the latter cause of termination is not equivalent to anchor failure, as conditions beyond this numerical instability could not be captured.

The occurrence of breakaway is identified in the images shown in Fig. 15. For low sustained loading levels $\left(F_{\text {sus }} / F_{\max } \leq 0 \cdot 6\right)$, full consolidation was achieved in a single calculation step, and Figs 15(a)-15(c) show anchor-soil boundaries at $90 \%$ consolidation. At $90 \%$ consolidation under sustained loading, $F_{\text {sus }} / F_{\max }=0.3$ (Fig. 15(a)) and $F_{\text {sus }} / F_{\max }=0.45$ (Fig. 15(b)), the soil remains in contact with the base of the anchor. As $F_{\text {sus }} / F_{\max }$ increases to $0 \cdot 6$, breakaway is observed at the anchor base, as evident from the development of a gap in Fig. 15(c). For simulations with $F_{\text {sus }} / F_{\text {max }} \geq 0 \cdot 7$, the calculations terminated due to severe mesh distortion during the course of consolidation, and the corresponding images, Figs 15(d) and 15(e), correspond to the end of numerically achievable consolidation (at $U=85 \%$ and $19 \%$ for $F_{\text {sus }} / F_{\max }=0.7$ and 0.84 , respectively, for the soil above the anchor). As expected, images Figs 15(a)-15(c) are consistent with observations from the centrifuge tests, showing that at low levels of sustained loading a gap does not form, whereas at higher sustained load levels a gap may form. Images shown in Figs 15(d) and 15(e) are slightly different, showing the development of a small gap at the edge of the anchor. These two instances correspond to levels of sustained load that can be regarded as a transition from a metastable condition with breakaway to failure without breakaway. Calculation termination due to severe mesh distortion prevented further investigation on this aspect. In addition to the cases where breakaway does or does not occur, Fig. 16 shows the development of total and effective stresses and pore water pressures during dimensionless time $T=0.8$ of consolidation, along the centreline of the interface at the base of the plate, for a no-breakaway case $\left(F_{\text {sus }} / F_{\max }=30 \%\right)$ and a breakaway case $\left(F_{\text {sus }} / F_{\max }=60 \%\right)$. The numerically determined evolution of stresses and pore water pressures is consistent with the hypothesis presented in Fig. 12, showing that breakaway may occur only when the effective stress at the base of the plate reaches zero. It is noteworthy that $\sigma_{\mathrm{v}}$ increases throughout consolidation in the breakaway case, whereas it remains constant in the no-breakaway case. This is because $\sigma_{\mathrm{v}}^{\prime}$ remains nil after breakaway, whereas $u$ increases owing to the dissipation of negative excess pore pressure. As a result, the total stress, $\sigma_{\mathrm{v}}=u+\sigma_{\mathrm{v}}^{\prime}$, varies in the same proportion as $u$.

\section{CONCLUDING REMARKS}

This paper reports results from both centrifuge tests and LDFE analyses that investigate the performance of plate anchors in normally consolidated clay under sustained loading. The soil deformation mechanisms around the anchor were established for sustained uplift at different ratios of the monotonic capacity. The focus of the work has been on identifying conditions under which sustained 


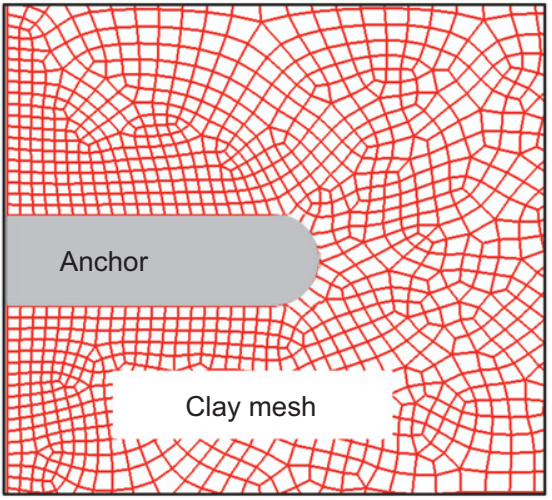

(a)

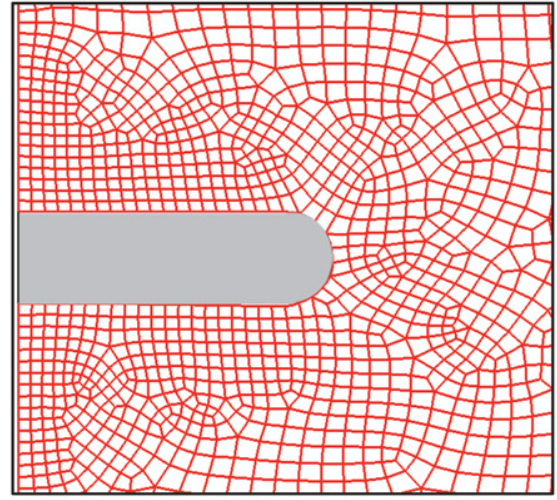

(b)

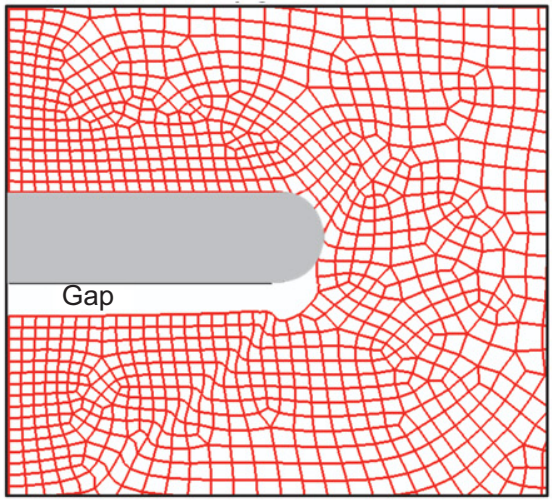

(c)

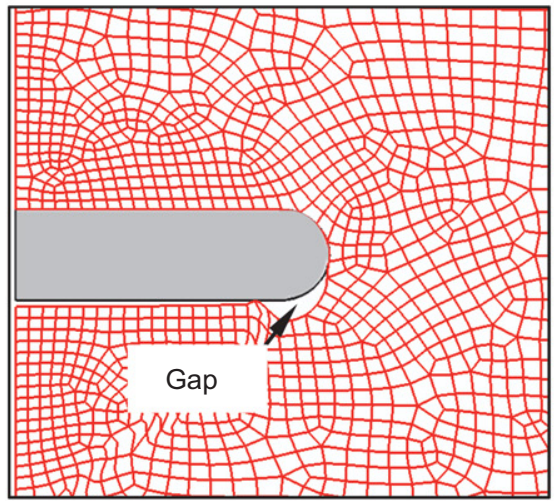

(d)

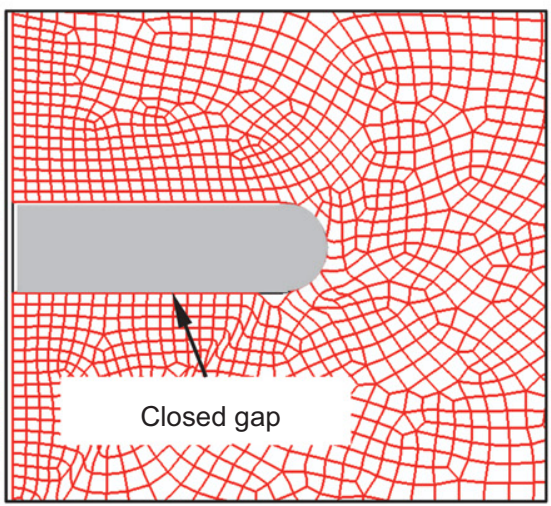

(e)

Fig. 15. Anchor soil boundaries established from $\mathrm{FE}$ analyses under different values of $\boldsymbol{F}_{\text {sus }} / \boldsymbol{F}_{\text {max }}$ and at different degrees of consolidation: (a) $F_{\text {sus }} I F_{\max }=0.3, U=90 \%$; (b) $F_{\text {sus }} I F_{\text {max }}=0.45, U=90 \% ;\left(\right.$ c) $F_{\text {sus }} I F_{\max }=0.60, U=90 \% ;(d) F_{\text {sus }} I F_{\text {max }}=0.70, U=85 \% ;\left(\right.$ e) $F_{\text {sus }} I F_{\text {max }}=0.84$, $U=19 \%$

loading may lead to failure and whether the soil at the base of the plate breaks away.

- Under low sustained load (lower than $60 \%$ of the ultimate monotonic capacity), the anchor remains stable throughout consolidation with small displacements and low velocities. The anchor-soil interface is fully bonded and the anchor capacity after maintaining the sustained load is increased due to strength enhancement through consolidation.

- For high levels of sustained loading (beyond $88 \%$ of the ultimate monotonic capacity), the anchor experiences significant displacement at velocities generating undrained behaviour. The anchor-soil interface remains fully bonded as negative excess pore pressures (suction) are continuously generated at the base of the anchor. The anchor fails by displacing into soil (above the initial anchor position) exhibiting lower strength that can no longer sustain the level of loading applied.

- For intermediate levels of sustained loading (between 60 and $88 \%$ of the ultimate monotonic capacity), breakaway at the base of the anchor occurs during the consolidation process as the vertical effective stresses reaches zero and suction can no longer be sustained. However, this does not lead to anchor failure as the reduction in capacity due to the change in failure mechanism is compensated by an increase in capacity at the front of the anchor. The latter is attributable to: $(a)$ strength enhancement during consolidation and $(b)$ reduced anchor velocities that lead to mobilisation of higher partially drained or drained soil strength.

\section{ACKNOWLEDGEMENTS}

The study forms part of the activities of the Australian Research Council Centre of Excellence for Geotechnical Science and Engineering and the Lloyd's Register Foundation (LRF) Centre of Excellence. LRF helps to protect life and property by supporting engineering-related education, public engagement and the application of research. Sponsorship of this work by the Australia China Natural Gas Technology Partnership Fund is also gratefully acknowledged. The fifth author is the recipient of an Australian Research Council Laureate Fellowship (project number FL130100059).

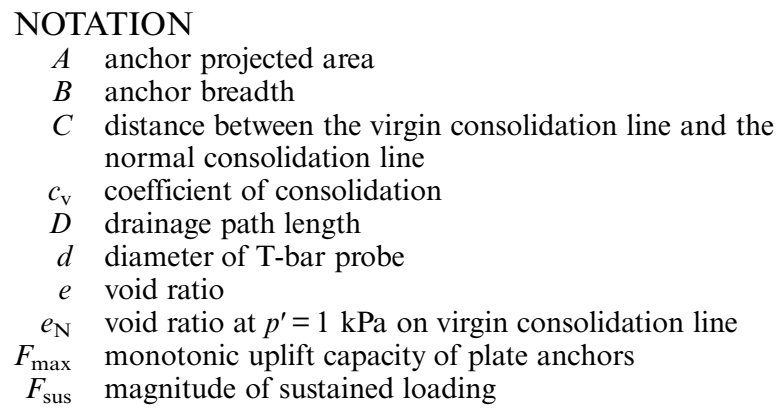




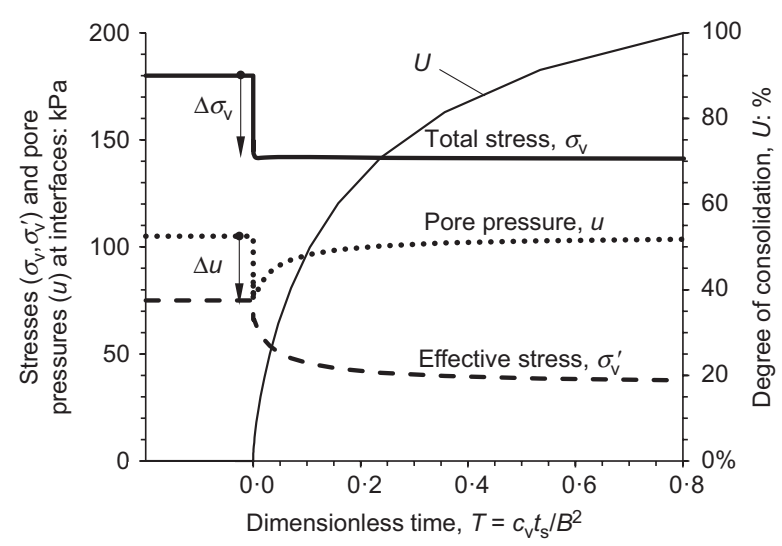

(a)

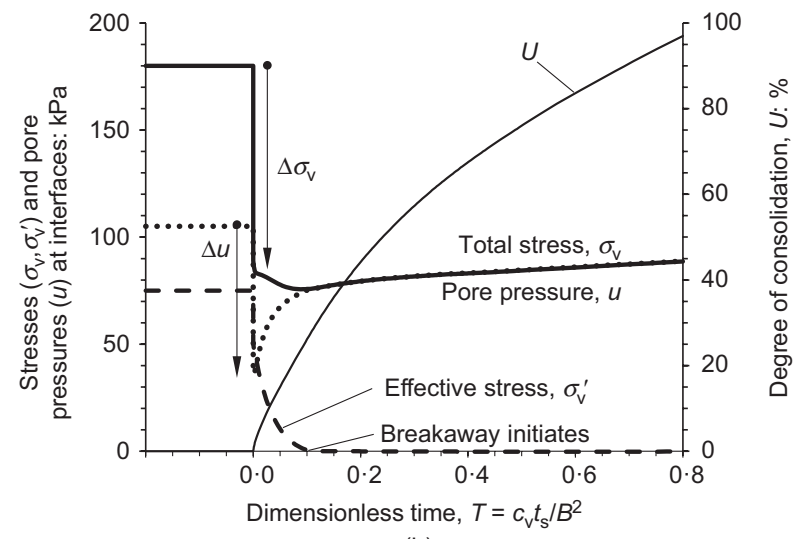

(b)

Fig. 16. Finite-element results showing the development of stresses $\left(\sigma_{\mathrm{v}}\right.$ and $\left.\sigma_{\mathrm{v}}^{\prime}\right)$ and pore pressures $(u)$ throughout sustained loading test along centreline of plate bottom interfaces at: (a) $F_{\text {sus }} / F_{\max }=30 \%$; (b) $F_{\text {sus }} / F_{\text {max }}=60 \%$

$K_{0} \quad$ coefficient of earth pressure

$k$ permeability of clay

$L$ anchor length

$M$ slope of critical state line in $q-p^{\prime}$ space

$N_{\mathrm{c}} \quad$ undrained bearing capacity factor

$s_{\mathrm{u}}$ undrained shear strength of clay

$T$ non-dimensional consolidation time during sustained loading

$t$ anchor thickness

$t_{\mathrm{s}} \quad$ consolidation time during sustained loading

$U$ degree of consolidation

$u$ pore water pressure

$V$ dimensionless anchor velocity

$v$ anchor velocity

$w$ anchor displacement

$w_{\mathrm{f}}$ anchor displacement at monotonic undrained failure

$w_{\mathrm{p}}$ targeted anchor displacement at start of sustained loading

$w_{\mathrm{s}}$ anchor displacement during sustained loading

$x$ horizontal distance from plate centre

$z$ embedment depth

$z_{\mathrm{i}} \quad$ initial embedment depth

$\gamma^{\prime} \quad$ effective unit weight of clay

$\gamma_{\mathrm{w}}$ unit weight of water

$\kappa$ slope of swelling line

$\lambda$ slope of virgin consolidation line

$\sigma_{\mathrm{v}} \quad$ vertical total stress

$\sigma_{\mathrm{v}}^{\prime} \quad$ vertical effective stress

$v$ Poisson ratio

$\phi^{\prime} \quad$ internal friction angle

\section{REFERENCES}

Cassidy, M. J., Gaudin, C., Randolph, M. F., Wong, P. C., Wang, D. \& Tian, Y. (2012). A plasticity model to assess the keying of plate anchors. Géotechnique 62, No. 9, 825-836, http://dx.doi.org/ 10.1680/geot.12.OG.009.

Chen, Z., Tho, K. K., Leung, C. F. \& Chow, Y. K. (2013). Influence of overburden pressure and soil rigidity on uplift behavior of square plate anchor in uniform clay. Comput. Geotech. 52, 71-81.

Colreavy, C., O’Loughlin, C. D. \& Randolph, M. F. (2016). Experience with a new dual pore pressure element piezoball. Int. J. Phys. Modelling Geotech., http://dx.doi.org/10.1680/ jphmg.15.00011.

Das, B. M. \& Singh, G. (1994). Uplift capacity of plate anchors in clay. Proceedings of the 4th international offshore and polar engineering conference, ISOPE, Golden, CO, USA, vol. I, pp. $436-442$.

DNV (Det Norske Veritas) (2002). DNV-RP-E302: Recommended practices: design and installation of plate anchors in clay. Oslo, Norway: DNV.

Elkhatib, S. \& Randolph, M. F. (2005). The effect of interface friction on the performance of drag-in plate anchors. In Frontiers in offshore geotechnics (eds S. Gourvenec and M. Cassidy), pp. 171-177. Boca Raton, FL, USA: CRC Press.

Garnier, J., Gaudin, C., Springman, S. M., Culligan, P. J., Goodings, D. J., Konig, D., Kutter, B. L., Phillips, R., Randolph, M. F. \& Thorel, L. (2007). Catalogue of scaling laws and similitude questions in geotechnical centrifuge modelling. Int. J. Phys. Modelling Geotech. 7, No. 3, 1-23.

Gaudin, C., O'Loughlin, C. D., Randolph, M. F. \& Lowmass, A. C. (2006). Influence of the installation process on the performance of suction embedded plate anchors. Géotechnique 56, No. 6, 381-391, http://dx.doi.org/10.1680/geot.2006.56.6.381.

House, A. R., Oliveira, J. R. M. S. \& Randolph, M. F. (2001). Evaluating the coefficient of consolidation using penetration tests. Int. J. Phys. Modelling Geotech. 1, No. 3, 17-25.

Hu, Y. \& Randolph, M. F. (1998). A practical numerical approach for large deformation problems in soil. Int. J. Numer. Analyt. Methods Geomech. 22, No. 5, 327-350.

Lehane, B., O'Loughlin, C., Gaudin, C. \& Randolph, M. F. (2009). Rate effects on penetrometer resistance in kaolin. Géotechnique 59, No. 1, 41-52, http://dx.doi.org/10.1680/geot.2007.00072.

Mahmoodzadeh, H., Randolph, M. F. \& Wang, D. (2014). Numerical simulation of piezocone dissipation test in clays. Géotechnique 64, No. 8, 657-666, http://dx.doi.org/10.1680/ geot.14.P.011.

Mahmoodzadeh, H., Wang, D. \& Randolph, M. F. (2015). Interpretation of piezoball dissipation testing in clay. Géotechnique 65, No. 10, 831-842, http://dx.doi.org/10.1680/ jgeot.14.P.213.

Mana, D. S., Gourvenec, S. M., Randolph, M. F. \& Hossain, M. S. (2012). Failure mechanisms of skirted foundations in uplift and compression. Int. J. Phys. Modelling Geotech. 12, No. 2, 47-62.

Martin, C. M. \& Randolph, M. F. (2001). Applications of the lower and upper bound theorems of plasticity to collapse of circular foundations. In Computer methods and advances in geomechanics (eds C. S. Desai, T. Kundu, S. Harpalani, D. Contractor and J. Kemeny), vol. 2, pp. 1417-1428. Rotterdam, the Netherlands: Balkema.

Merifield, R., Sloan, S. \& Yu, H. (2001). Stability of plate anchors in undrained clay. Géotechnique 51, No. 2, 141-154, http://dx.doi. org/10.1680/geot.2001.51.2.141.

O'Loughlin, C. D. Blake, A. Richardson, M. D. Randolph, M. F. \& Gaudin, C. (2014). Installation and capacity of dynamically embedded plate anchors as assessed through centrifuge tests. Ocean Engng 88, 204-213.

O’Neill, M. P., Bransby, M. F. \& Randolph, M. F. (2003). Drag anchor fluke-soil interaction in clays. Can. Geotech. J. 40, No. 1, 78-94.

Randolph, M. F. \& Hope, S. (2004). Effect of cone velocity on cone resistance and excess pore pressures. Proceedings of the international symposium on engineering practice and performance of soft deposits, Osaka, Japan, pp. 147-152.

Randolph, M. F., Jewell, R. J., Stone, K. J. L. \& Brown, T. A. (1991). Establishing a new centrifuge facility. In Centrifuge 91: proceedings of the international conference on geotechnical centrifuge modelling (eds H.-Y. Ko and F. G. McLean), pp. 3-9. Rotterdam, the Netherlands: Balkema. 
Richardson, M. D., O’Loughlin, C. D., Randolph, M. F. \& Gaudin, C. (2009). Setup following installation of dynamic anchors in normally consolidated clay. J. Geotech. Geoenviron. Engng, ASCE 135, No. 4, 487-496.

Rowe, R. K. (1978). Soil structure interaction analysis and its application to the prediction of anchor plate behaviour. $\mathrm{PhD}$ thesis, University of Sydney, Sydney, Australia.

Rowe, R. K. \& Davis, E. H. (1982). The behaviour of anchor plates in clay. Géotechnique 32, No. 1, 9-23, http://dx.doi.org/10.1680/ geot.1982.32.1.9.

Song, Z., Hu, Y. \& Randolph, M. F. (2008). Numerical simulation of vertical pullout of plate anchors in clay. J. Geotech. Geoenviron. Engng, ASCE 134, No. 6, 866-875.

Stanier, S. A. \& White, D. J. (2013). Improved image-based deformation measurement in the centrifuge environment. Geotech. Testing J. 36, No. 6, 915-928.

Stanier, S. A., Ragni, R., Bienen, B. \& Cassidy, M. J. (2014). Observing the effects of sustained loading on a spudcan footing in clay. Géotechnique 64, No. 11, 918-926, http://dx.doi.org/ 10.1680/geot.14.P.003.

Stewart, D. P. (1992). Lateral loading of piled bridge abutments due to embankment construction. PhD thesis, Department of Civil and Environmental Engineering, The University of Western Australia, Crawley, WA, Australia.

Stewart, D. P. \& Randolph, M. F. (1994). T-Bar penetration testing in soft clay. J. Geotech. Engng, ASCE 120, No. 12, 2230-2235.

Teh, K. L., Cassidy, M. J., Leung, C. F., Chow, Y. K., Randolph, M. F. \& Quah, C. K. (2008). Revealing the bearing failure mechanisms of a penetrating spudcan through sand overlying clay. Géotechnique 58, No. 10, 793-804, http://dx.doi.org/ 10.1680/geot.2008.58.10.793.

Tho, K. K., Chen, Z., Leung, C. F. \& Chow, Y. K. (2013). Pullout behaviour of plate anchor in clay with linearly increasing strength. Can. Geotech. J. 51, No. 1, 92-102.

Thorne, C. P., Wang, C. X. \& Carter, J. P. (2004). Uplift capacity of rapidly loaded strip anchors in uniform strength clay. Géotechnique 54, No. 8, 507-517, http://dx.doi.org/10.1680/ geot.2004.54.8.507.

Tian, Y., Gaudin, C., Randolph, M. F. \& Cassidy, M. J. (2014). Influence of padeye offset on bearing capacity of three-dimensional plate anchors. Can. Geotech. J. 52, No. 6, 682-693.

Wang, D. \& O’Loughlin, C. D. (2014). Numerical study of pull-out capacities of dynamically embedded plate anchors. Can. Geotech. J. 51, No. 11, 1263-1272.

Wang, D., Hu, Y. \& Randolph, M. F. (2010). Three-dimensional large deformation finite-element analysis of plate anchors in uniform clay. J. Geotech. Geoenviron. Engng, ASCE 136, No. 2, $355-365$.

Wang, D., Hu, Y. \& Randolph, M. F. (2011). Keying of rectangular plate anchors in normally consolidated clays. J. Geotech. Geoenviron. Engng, ASCE 137, No. 12, 1244-1253.

Wang, D., Gaudin, C. \& Randolph, M. F. (2013). Large deformation finite element analysis investigating the performance of anchor keying flap. Ocean Engng 59, 107-116.

Wang, D., Bienen, B., Nazem, M., Tian, Y., Zheng, J., Pucker, T. \& Randolph, M. F. (2015). Large deformation finite element analyses in geotechnical engineering. Comput. Geomech. 65, 104-114.

White, D. J., Take, W. A. \& Bolton, M. D. (2003). Soil deformation measurement using particle image velocimetry (PIV) and photogrammetry. Géotechnique 53, No. 7, 619-631, http://dx.doi.org/ 10.1680/geot.2003.53.7.619.

Wilde, B., Treu, H. \& Fulton, T. (2001). Field testing of suction embedded plate anchors. Proceedings of the 11th international offshore and polar engineering conference, Stavanger, Norway, vol. 2, pp. 544-551.

Wong, P., Gaudin, C., Randolph, M. F., Cassidy, M. J. \& Tian, Y. (2012). Performance of suction embedded plate anchors in permanent mooring applications. Proceedings of the 22nd international offshore and polar engineering conference, Rhodes, Greece, vol. 2, pp. 640-645.

Wroth, C. P. (1984). The interpretation of insitu soil tests. Géotechnique 34, No. 4, 449-489, http://dx.doi.org/10.1680/ geot.1984.34.4.449.

Yang, M., Murff, J. D. \& Aubeny, C. P. (2010). Undrained capacity of plate anchors under general loading. J. Geotech. Geoenviron. Engng, ASCE 136, No. 10, 1383-1393.

Yang, M., Aubeny, C. P. \& Murff, J. D. (2012). Behaviour of suction embedded plate anchors during the keying process. J. Geotech. Geoenviron. Engng, ASCE 138, No. 2, 174-183. 